\title{
Miranda
}

Revue pluridisciplinaire du monde anglophone /

Multidisciplinary peer-reviewed journal on the English-

speaking world

$12 \mid 2016$

Mapping gender. Old images ; new figures

\section{L'action en noir et au féminin dans New York Taxi (Tim Story, 2004)}

\section{Hélène Charlery}

URL : http://journals.openedition.org/miranda/8552

DOI : 10.4000/miranda.8552

ISSN : 2108-6559

\section{Éditeur}

Université Toulouse - Jean Jaurès

\section{Référence électronique}

Hélène Charlery, «L'action en noir et au féminin dans New York Taxi (Tim Story, 2004) 》, Miranda [En ligne], 12 | 2016, mis en ligne le 02 mars 2016, consulté le 16 février 2021. URL : http:// journals.openedition.org/miranda/8552 ; DOI : https://doi.org/10.4000/miranda.8552

Ce document a été généré automatiquement le 16 février 2021.

\section{(c) (i) () $\Theta$}

Miranda is licensed under a Creative Commons Attribution-NonCommercial-NoDerivatives 4.0 International License. 


\title{
L'action en noir et au féminin dans New York Taxi (Tim Story, 2004)
}

\author{
Hélène Charlery
}

1 Le film Taxi, réalisé par Gérard Pirès, écrit et produit par Luc Besson, sort sur les écrans français en 1998. Il réunit Daniel Morales (Samy Naceri), un livreur de pizza qui obtient sa licence de taxi au début du film, et Emilien Coutant-Kerbalec (Frédéric Diefenthal), un inspecteur de la police marseillaise. Alors que Daniel est considéré comme un véritable as du volant, l'inspecteur Coutant-Kerbalec est incapable de conduire une voiture et d'obtenir son permis. Il est alors contraint de faire appel au service de Daniel Morales et de sa Peugeot 406 customisée, afin de déjouer un gang de braqueurs de banque allemands qui, aux volants de Mercedes surpuissantes, échappent à la police marseillaise. Le succès du film Taxi repose sur le nombre de scènes de poursuite de bolides vrombissants dans les rues de Marseille, rythmées par la musique d'Akhenaton, du groupe IAM. ${ }^{1}$ Dans le film, la vitesse est associée à un discours sur la masculinité et sur la francité que j'analyserai dans la dernière partie de cet article.

2 Cinq ans après la sortie du film français, son remake, New York Taxi (Tim Story), sort sur les écrans américains. ${ }^{2}$ Le remake reprend le scénario du film français. Dans la version américaine, l'humoriste blanc Jimmy Fallon tient le rôle d'Émilien Coutant-Kerbalec qui devient Andy Washburn, mais les braqueurs de banque allemands sont transformés en braqueuses brésiliennes dont le chef de file, Vanessa, est interprété par le mannequin brésilien, Giselle Bündchen. Plus intéressant encore, l'acteur français d'origine algérienne Samy Naceri, qui incarne Daniel Morales dans le film français, est remplacé par la productrice, actrice et chanteuse de hip hop noire américaine Queen Latifah qui interprète le rôle de Belle Williams. Alors que dans la comédie d'action française Taxi, les personnages féminins sont relégués à la fonction d'accessoires, comme nous le verrons dans cet article, dans la version américaine, la maitrise de l'engin motorisé est le fait exclusif des personnages féminins. Dans ses notes de production la Twentieth Century Fox, qui produit et distribue le film, décrit le remake américain comme une "comédie d'action déconstruite, fondée sur l'inversion des rôles sexuels». Le commentaire implique que la Fox s'enorgueillit de cette touche spécifique apportée à la 
version américaine. Mais, il implique également que la société de production et de distribution du film fait de la maitrise de la vitesse motorisée un marqueur implicite de la masculinité.

3 Ainsi, la version américaine délaisse le discours du film français sur l'identité nationale mais, par l'inversion des rôles sexuels et le choix d'une distribution interraciale, associe également la vitesse à un discours de genre et de race, puisque les as du volant du remake sont des femmes étrangères ou non-blanches, et le policier maladroit est un homme blanc. A l'instar du studio de production, l'actrice noire Queen Latifah place son rôle dans cette comédie d'action dans une perspective de genre :

The script had a great combination of action and comedy [...]. But, I really took it on because I like being physical in movies. Taxi gave me the opportunity to drive and do stunts and have fun. I have that $\mathrm{X}$ gene in me, the one that makes you willing to jump out of planes, ride motorcycles and do karate. I was really looking forward to doing that kind of movie and finally Taxi came along (62).

Si l'actrice occulte la dimension ethnique et raciale du film, elle n'échappe pas au critique du New York Times, Dave Kehr, comme le montre son analyse plus acerbe du remake américain :

It's interesting - and a bit depressing - to see how easily one country's ethnic clichés can be superimposed on another's. Here, Queen Latifah has been drafted (as she has been drafted many times before) to represent some kind of irrepressible life force, supposedly unavailable to uptight white folks. Her bursting ethnicity somehow liberates Mr. Fallon's character, a timid young man who lives next door to his alcoholic mother [...]. By the end of the film, Washburn has been transformed into a dare-devil driver in his own right [...].

5 Comme le résume le critique du journal, l'objet de New York Taxi est la transformation de la masculinité de ce personnage masculin blanc, d'abord présentée comme attachante, certes, mais faillible, avant qu'elle ne devienne conquérante. Par son inversion des rôles sexuels, propre au genre de la comédie, et son casting d'un duo d'acteurs noir et blanc, New York Taxi permet un bouleversement temporaire des rôles sexuels et raciaux, tout en garantissant un retour à la normal sécurisant pour les spectateurs grand public qui composent le groupe-cible des producteurs.

Contrairement à ce que l'actrice Queen Latifah laisse entendre dans ses commentaires sur le film, l'action de la version américaine de Taxi n'inclut pas de scène de combat ni de saut d'avion et encore moins de course de motos. L'action du film New York Taxi se manifeste principalement par la vitesse d'engins motorisés, lancés à travers les rues de Manhattan, dans le hall bondé de Grand Central Station ou dans tout autre lieu où il est difficile d'imaginer un véhicule, qui plus est en mouvement. L'objet de cet article est de déterminer la façon dont l'utilisation de la vitesse par les personnages féminins de New York Taxi participe à la transformation de la masculinité blanche, seul véritable objet du film. On se demandera alors si, en dépit du fait qu'ils soient au cœur de l'action/vitesse, les personnages féminins du film n'occupent-ils pas, comme l'écrit Geneviève Sellier, « la place traditionnelle dévolue aux femmes dans la culture occidentale : non pas sujet de l'histoire et du récit, mais objet [...] pour un sujet masculin » (16).

Il s'agira dans un premier temps d'analyser les scènes de présentation des deux personnages féminins qui ont recours à la vitesse dans le film. Nous confronterons la présentation de ces personnages aux héroïnes d'action contemporaines et à leur théorisation dans les travaux de spécialistes du genre. Cette première analyse mettra en exergue une distinction dans les représentations du corps des braqueuses 
brésiliennes et de celui de l'actrice noire américaine. Nous verrons également que la vitesse, implicitement marquée comme un signifiant masculin dans le film, nécessite une justification narrative de sa maîtrise par les personnages féminins et de cette absence de maitrise chez le personnage masculin blanc. La deuxième partie de cet article portera sur la relation entre Belle Williams et Andy Washburn. Au-delà de la comédie d'action, le casting d'un duo d'acteurs noir et blanc permet un rapprochement avec le genre du buddy movie interracial, genre très populaire dans les années 1980 qui repose sur l'association improbable de deux personnages masculins, l'un blanc, l'autre noir, qu'en apparence tout oppose. Enfin, dans la dernière partie de cet article, je confronterai les discours de race et de genre du film New York Taxi au discours de la masculinité et de la francité de Taxi.

\section{Les corps féminins en mouvement dans New York Taxi : érotiser la vitesse féminine blanche, normaliser la vitesse féminine noire}

8 Comme l'explique Hilary Neroni dans The Violent Women : Femininity, Narrative and Violence in Contemporary American Cinema, les spécialistes de films violents et ceux qui analysent les représentations de la masculinité dans le cinéma ne remettent pas en question le lien entre l'action violente et la masculinité, si bien que l'analyse de l'utilisation de la violence par des personnages féminins se fait par rapport à un référent masculin (45-46). Il faut toutefois dire que le cinéma américain a politiquement inscrit l'action violente et la vitesse dans un discours sur la masculinité blanche. Dans les années 1980, le genre vise avant tout à faire l'apologie de ces héros qui assimilent les signifiants de l'action à ceux d'une hyper masculinité reaganienne (Neroni 46). Dans Hard Bodies: Hollywood Masculinity in the Reagan Era, paru en 1994, Susan Jeffords montre en effet que le film d'action met en scène des corps infatigables, musclés et invincibles, opposés à ces autres corps dits soft ou dévoyés (errant) qui illustrent des pratiques contraires aux valeurs conservatrices des politiques publiques et économiques de l'administration Reagan (24-25). Selon Jeffords, les caractéristiques esthétiques des films d'action sont la manifestation externe de cette masculinité blanche et américaine dite hard (Jeffords 1993, 245). Dans ces films, les protagonistes féminins sont exclus de cette dichotomie hard/soft, et servent plutôt d'accessoires à la trame narrative, dans laquelle l'action violente et spectaculaire reste principalement le fait d'un personnage masculin.

Quant à la vitesse, elle est également construite comme masculine au cinéma, comme le suggère Forman Murray :

Films that reproduce highway driving or cataclysmic collisions as predominantly male practices are entered into a gendered social "arena," merging with prevailing discourses of masculinity, virility, and power, all of which reinforce and extend an image of the human connection with cars as an especially masculine devotion. This does not eradicate the possibility of an empowering female association with cars, as Heart Like a Wheel (1983) and Thelma and Louise (1991) illustrate. Indeed, associating strong female characters with cars emerges as a strategy through which to both enunciate their autonomy and power and inscribe nuanced masculine identities onto the characters.

These exceptions notwithstanding, the prevailing imagery in our popular culture 
suggests that men are much more predisposed to particular practices and outcomes formed in and through their identifications with automobiles. [...]. The same can be said of the particular masculine formations that commonly cohere around cinematic portrayals of automobiles. For example, the image of an assertive and controlled masculinity that is traced onto Steve McQueen in Bullitt is pointedly linked to his car, as a vehicle connoting speed, thrust, and agility - each structuring his relentless pursuit of various goals and objectives (113-114).

Cette construction implicite entre masculinité et vitesse ou action violente et spectaculaire peut expliquer pourquoi les scènes d'ouverture de films d'action contemporains mettant en scène des personnages féminins prennent le temps et le soin de rationaliser, de justifier et de normaliser l'utilisation de l'action violente par des personnages féminins dépeints comme hors-normes. Les scènes d'ouverture de Charlie's Angels (McG, 2000) ou de Lara Croft : Tomb Raider (Simon West, 2001) sont des modèles du genre. Comme le précise la scène d'introduction de Charlie's Angels :

Once upon a time there were three very different little girls...They grew up to be three very different women. But they have three things in common: they're brilliant, they're beautiful...and they work for me...My name is Charlie.

11 Sur la voix-off de Charlie, on voit les «trois petites filles» et ce qui marque leur différence dès l'adolescence: Natalie (Cameron Diaz) fait des cascades pendant une leçon de conduite, Alex (Lucy Liu) gagne un concours de saut d'obstacles, et Dylan (Drew Barrymore) fume dans les toilettes d'une école défiant la caméra de sécurité d'un geste outrancier. Devenues femmes dans la même scène d'introduction, elles sont à nouveau singularisées : Natalie gagne une certaine somme d'argent à un jeu télévisé, Alex participe à un programme aérospatial et Dylan défie l'autorité militaire en assenant un violent coup de poing à son supérieur avant de quitter les rangs de l'armée. La scène d'introduction de Charlie's Angels construit donc la différence de ces personnages féminins qui vont construire et animer l'action du film. De même, dans Lara Croft: Tomb Raider, les spectateurs comprennent, par le comportement et la réaction de l'entourage masculin de l'aventurière, que la séance d'entraînement de cette dernière, qui ouvre de manière très spectaculaire le film, est une activité régulière de la jeune femme. En d'autres termes, ces scènes d'ouvertures visent à normaliser l'utilisation de l'action/vitesse par la construction de personnages féminins atypiques.

On trouve ce procédé de construction narrative dans la scène d'ouverture du film New York Taxi. La présentation de Belle Williams est intégrée au générique du film. On y voit le personnage maîtriser la vitesse, tout en étant structurée dans une identité de genre bien spécifique. Rythmées par le "Crazy in Love » de Beyoncé, les images en montage rapide alterné dévoilent plusieurs types de véhicules lourds, motorisés (train, métro, voiture, camion), tous principalement statiques, entre lesquels un cycliste traverse la ville à toute vitesse, y compris via les couloirs du métro. Les spectateurs ne savent pas encore que c'est Belle qui pilote ce vélo, mais la scène d'ouverture les prépare à la personnalité atypique du personnage en distinguant les sphères d'activité des genres selon un modèle actif/passif et consommation/travail et production. En effet, lorsqu'elle traverse les allées du grand magasin Macy's à vélo, ce sont des femmes, une cliente et la vendeuse, qui se parfument et consomment, mais ce sont des hommes que l'on voit principalement accomplir des tâches physiques et pénibles dans le cadre de leurs activités professionnelles : des blanchisseurs portant de sacs de vêtements sales aux ouvriers du bâtiment, manipulant des machines lourdes, en passant par le chauffeur du poids-lourd sur lequel son vélo rebondit. La scène se conclut lorsque 
l'engin pénètre dans l'entreprise de livraison rapide dans laquelle Belle Williams travaillait jusqu'alors, et où l'attendent ses collègues, tous masculins. Les spectateurs découvrent alors que c'est bien Belle qui traversait la ville sur son vélo. La présence très masculine autour du personnage féminin cautionne sa maîtrise de la vitesse et son occupation de l'espace urbain dans le cadre de son activité professionnelle. Le personnage est donc placé dans ce modèle actif de production qui a été construit dans le générique comme une sphère principalement occupée par des hommes.

Dans la scène qui suit, c'est Belle elle-même qui, dans ses propos, place son corps féminin en objet érotisé du regard masculin. En effet, après avoir à nouveau battu un record de vitesse, s'adressant à l'ensemble des collègues qui l'entourent, elle se décrit comme une "fille» (" the girl»), objet du regard et du plaisir masculin: "What you gonna do with the girl but love her and pay her. Give me my money. [...] I just stayed ahead of you because I know you enjoyed the view ». Ici, la vitesse («I just stayed ahead of you ») est associée à une stratégie d'exhibition et de contemplation érotique («because I know you enjoyed the view»). Son patron la félicite pour son sérieux professionnel, en la replaçant dans une ambigüité sexuelle ("You're the best man I've ever had»), avant de lui offrir une énorme boîte rose ("This is for you, girl»), qui contient un turbocompresseur en titane pour gonfler la puissance du moteur de son taxi. La réaction de surprise de Belle à la vue du cadeau («It's just my size, too») entretient l'ambigüité de la présentation de l'identité de genre du personnage féminin par l'association de références à l'hyper-féminisation de la boîte et le caractère fonctionnel de son contenu. On retrouve cette association lorsque Belle, vêtue d'une nuisette rose, installe le nouveau turbocompresseur dans sa voiture. Dans la scène d'introduction, l'arrivée du compagnon de Belle, Jesse, un ouvrier en bâtiment, grand et particulièrement musclé, vient lever toute ambiguïté sur l'orientation sexuelle du personnage, par une mise en exergue de son hétérosexualité et du couple qu'elle forme avec un homme qui est son exact opposé. En effet, contrairement à Belle, Jesse ne montre aucun intérêt pour les voitures et la mécanique, et toutes ses interventions dans le film visent à tenter de la demander en mariage. Cette scène d'ouverture associe donc la maîtrise de la vitesse mécanique et de l'espace urbain par le personnage principal féminin du film à la construction très spécifique de son identité de genre. Belle est une femme, mais une femme bagarreuse ( « feisty ») et puissante, comme elle se décrit elle-même dans le film.

Tico Romao note que dans les années 1980, l'expression de la masculinité reaganienne, mentionnée précédemment, a donné la priorité à la force du combat physique et au maniement des armes lourdes au détriment de l'agilité au volant et de la maîtrise de la vitesse dans l'espace urbain (144). Ceci peut expliquer pourquoi les travaux de recherche sur les représentations de la féminité et de la masculinité dans les films d'action mettent peu l'accent sur la notion de vitesse motorisée, pourtant intrinsèque à la violence spéculaire de ce genre de films. La priorité des travaux de recherche est alors donnée à la façon dont l'action spectaculaire est transposée au féminin (blanc) et à la manière dont elle modifie les corps féminins et leur évolution dans l'espace diégétique.

Raphaëlle Moine constate qu'un « nouveau mode d'engagement du corps féminin dans le spectacle cinématographique » américain naît dans les années 1990 (Moine 2010, 8). ${ }^{3}$ Ce nouveau mode amène une modification du corps des héroïnes. L'évolution du personnage de Sarah Connor et la transformation du corps musculeux de l'actrice Linda 
Hamilton, de Terminator (James Cameron, 1984) à Terminator 2: Judgment Day (James Cameron, 1991), illustrent le glissement du statut du personnage féminin : il passe du rôle d'accessoire au lancement d'une action strictement masculine à une participation plus active à la dimension spectaculaire du film d'action. Les travaux récents menés sur la transformation des corps féminins des héroïnes d'action des années 2000 mènent à l'émergence de concepts tels que babes in arms, babes in boots ou autres fashionistas, visant à théoriser ces nouveaux corps que l'on voit dans les films comme Charlie's Angels ou Lara Croft, mentionnés précédemment, mais également dans Matrix (Andy et Lana Wachowski, 1999) ou Kill Bill-I (Quentin Tarantino, 2003). Bien que suggérant une infantilisation et objectification du personnage féminin (babes, -istas), ces concepts et expressions révèlent l'arrivée, aux côtés d'une féminité portée par un corps bodybuildé (comme celui de l'actrice Linda Hamilton), d'une construction plus hybride associant une « masculinité agressive et active » à une " féminité glamour » (Moine 29-31), visible dans les formes du corps et les costumes des actrices à l'affiche de ces films. Les héroïnes sont au cœur du spectacle de l'action violente. Elles manient les armes lourdes aussi bien que les rudiments du combat physique, et peuvent recevoir des coups violents ou des balles (in arms, in boots). Toutefois, l'utilisation du ralenti et les costumes plus moulants mettent en exergue l'élasticité, la légèreté et la grâce du mouvement des corps féminins, très souvent morcelés par les gros plans ou les angles de caméra. Ces procédés techniques et la modification des corps plus élancés des héroïnes amènent Marc O'Day à commenter ces formes hybrides et la position duelle des héroïnes. Elles sont à la fois sujet actif, car elles participent directement à l'action et à son spectacle, et objet sexuel, par la mise en scène de leur corps érotisé (216). La transformation physique et l'hyper féminisation de certaines héroïnes d'action dans les années 2000 (O’Brien 68) viendrait donc mettre en exergue une désirabilité hétérosexuelle qui compenserait la mise en scène de ces héroïnes dans des situations filmiques traditionnellement associées à des personnages masculins depuis les années 1980 . Jeffrey Brown apporte une nuance à cette dichotomie, avançant l'idée d'une fonctionnalité différente du corps féminin de l'héroïne d'action :

Certainly the action heroine is often filmed to accentuate her body, but this new hard body is not offered up as a mere sexual commodity. While the well-toned muscular female body is obviously an ideal in this age of physical fitness, it is presented in these films as first and foremost a functional body, a weapon (25).

Toutefois, dans New York Taxi, le corps de l'actrice Queen Latifah n'est pas musculeux. La force du personnage qu'elle interprète tient à sa maîtrise du volant et la caméra n'insiste pas sur sa silhouette. C'est en cela que le traitement filmique du personnage interprété par l'actrice noire est différent de celui du personnage qu'incarne le mannequin brésilien Giselle Bündchen. ${ }^{4}$ La présentation de Vanessa, le chef de file des braqueuses brésiliennes, suit l'analyse de Marc O’Day et le schéma démonstratif explicité par Precilla Choi dans le cadre du sport :

Because the visibility of a woman [...] demonstrating strength, speed and agility is more consistent with traditional notions of masculinity, we need to be assured of the sportswoman's femininity, hence the emphasis on beauty and heterosexual desirability (9).

Les corps des braqueuses brésiliennes, courtement vêtus, sont dévoilés à l'écran, offrant le spectacle d'une féminité " glamour » et sulfureuse, avant qu'elles ne soient intégrées à l'action du film et qu'on ne les voie, fusils au poing, braquer la première banque, et avant que le spectateur ne découvre Vanessa sillonnant les rues de New 
York au volant d'une BMW. Alors que le rythme rapide du montage avait marqué la présentation de Belle Williams, c'est le ralenti qui est choisi pour présenter Vanessa. Rythmés par la musique très suggestive de "Wild Thing ", interprété par Tone Loc, le ralenti et le mouvement vertical de la caméra dévoilent ses longues jambes, devant les portes de l'aéroport, jusqu'à un plan plus élargi qui place les quatre braqueuses au centre de l'écran, sous le regard très satisfait de policiers en patrouille, à qui Vanessa envoie un baiser. Bien que le plan de la caméra permette d'intégrer toutes les braqueuses dans le champ, c'est bien les mouvements du corps de Vanessa, que la caméra suit au ralenti. Comme mentionné précédemment, ce personnage en particulier reprend la dualité des héroïnes des récents films d'actions : au volant de la BMW et en tant que chef de file du gang, ce personnage est bien un sujet actif qui initient les courses poursuites du film. Les plans et les mouvements de la caméra dévoilant les parties, très souvent dénudés, du corps du personnage, sous le regard de spectateurs intra-diégétiques masculins, en font également un objet érotisé du regard. Les champscontre-champs entre le corps de Vanessa et ces personnages masculins reproduisent les trois sources du regard/gaze (caméra, personnages et spectateurs), identifiées par Laura Mulvey dans son article "Visual Pleasure and Narrative Cinema" (1975). La réaction de satisfaction des personnages masculins, témoins de l'exhibition du corps de Vanessa, vient soutenir, par mimétisme, l'approbation des spectateurs dans le plaisir visuel qu'ils pourraient prendre à observer attentivement (du fait de l'échelle des plans ou du ralenti) le corps du personnage.

On note que même Belle, pourtant aguerri à la vitesse motorisée, est surprise non seulement de découvrir Vanessa au volant de la BMW, mais surtout de la voir maittriser aussi bien la vitesse que les freinages d'urgence dans les espaces exigus de la ville. Si le film prend le temps de justifier sur le plan narratif l'engouement de Belle pour la mécanique et la vitesse, dans le cas de Vanessa, seule sa plastique est mise en exergue, sans pour autant l'exclure de l'action du film. Il n'en reste pas moins que dans New York Taxi la maîtrise de la vitesse motorisée est associée aux personnages féminins, et à l'Autre ou au non-blanc. Ces personnages féminins qui contrôlent cette vitesse avec maestria placent le cadre dans laquelle va s'inscrire la présentation du personnage masculin blanc, marquée par la caractérisation d'une masculinité blanche défaillante.

\section{La dimension masculine de la vitesse à l'écran : le personnage féminin noir au service de la masculinité blanche}

19 Il ne faut pas longtemps aux spectateurs pour comprendre que le personnage d'Andy Washburn n'est pas un héros reaganien ou le hard body décrit par Susan Jeffords. Il est plutôt l'alter ego fragile des duos mis en scènes dans les buddy movies, définis précédemment. L'association de Daniel Morales et Emilien Coutant-Kerbelec dans le film Taxi n'est pas sans rappeler le duo incarné par Pierre Richard et Gérard Depardieu dans la trilogie à succès de Francis Veber, La Chèvre (1981), Les Compères (1983) et Les Fugitifs (1986), qui associe une force brute et virile à un personnage qui se distingue particulièrement par sa maladresse. Cette force brute et virile vient précisément en aide à cette masculinité maladroite. $\mathrm{Si}$, au début du film, tout semble opposer les deux personnages, ils finissent par unir leurs forces pour atteindre un objectif commun. L'association des protagonistes du buddy movie construit une solidarité masculine à 
laquelle les personnages féminins servent d'accessoires, quand ils n'en sont pas tout bonnement exclus. Bien qu'intégrant un personnage féminin dans le duo de protagonistes principaux, le film New York Taxi reprend certains aspects de cette association exclusivement masculine.

La force brute et virile est transposée dans la maîtrise de la vitesse dont Belle Williams fait preuve tout au long du film. La maladresse caractérise le personnage d'Andy Washburn, comme le montre sa scène d'introduction, marquée par le spectacle de l'action comique et du burlesque. Cette présentation intervient dans un environnement particulièrement ethnicisé. Les spectateurs de New York Taxi découvrent en effet Andy Washburn alors qu'il descend d'un bus qui le dépose dans un quartier racialisé, comme le montrent les figurants et les plans larges des décors de la scène. En descendant du bus, il dépose fièrement sur son visage une fausse moustache, censée apporter la dernière touche au costume cubain qu'il revêt afin de piéger des trafiquants de cartes téléphoniques prépayées originaires de La Havane. Le ressort comique de la scène tient au décalage entre la force et la maitrise de la situation qu'il souhaite mettre en œuvre en tant que personnage principal masculin du film, son arrivée en bus et son incapacité à véritablement incarner un immigrant cubain. L'arrestation qui suit est un véritable fiasco du fait de l'excès de zèle du personnage et de son incapacité à conduire : son partenaire est accidentellement blessé par une balle, les trafiquants cubains s'enfuient avec l'argent et les cartes prépayées au volant d'une Ford Mustang, et, en tentant de les poursuivre avec la voiture de son coéquipier, Andy Washburn confond la marche avant et la marche arrière et percute la façade d'un restaurant, devenant la risée de ses collègues de travail et de la presse locale. Dans cette scène d'introduction, la masculinité blanche défaillante d'Andy Washburn est caractérisée par son incapacité à conduire. Le personnage est loin d'incarner la masculinité virile qu'il tente d'imiter dans son costume d'emprunt. La scène suivante ne fait qu'entériner cette impuissance puisque Washburn est sévèrement secoué par sa supérieure hiérarchique et ancienne compagne, le lieutenant Robbins, interprétée par l'actrice hispanique Jennifer Esposito, qui finit par lui retirer son permis. Cette présentation du personnage masculin blanc, celles de Vanessa, la braqueuse brésilienne, et de Belle Williams, noire américaine, associent dans un premier temps l'action, la vitesse, l'autorité et l'agilité à l'ethnicité et aux personnages non-blancs ou non-Américains.

On pourrait alors y lire une construction contraire à la masculinité ethnique et raciale telles qu'elles ont été mises en scène dans les buddy movies interraciaux des années 1980 qui associaient l'action et le rire filmique à une ethnicité bien encadrée. En 1993, Ed Guerrero s'alarmait en effet du fait que, dans les buddy movies interraciaux de la décennie, les acteurs noirs étaient placés en tête d'affiche de films dans lesquels les personnages qu'ils interprétaient étaient plongés dans un univers entièrement blanc, complètement isolés de leur univers culturel et racial ou de la présence de tout autre personnage noir (Guerrero 237)..$^{5}$ Selon l'auteur, le genre faisait l'apologie de ce personnage noir qu'il qualifiait de «zéro déraciné, culturellement différencié par la simple couleur de peau " (ma traduction, 237). Il parlait alors de mise en «détention préventive » du personnage noir (protective custody, 239), puisqu'il partageait l'affiche avec un « chaperon culturel et idéologique » blanc (239). Sur le plan de la masculinité noire, la présence de ce chaperon blanc canalisait la sexualité potentielle des jeunes personnages noirs interprétés par Eddie Murphy, comme Alex Foley dans la série des Flics de Beverly Hills ou Reggie Hammond dans 24 Hours, ou servait également à des 
personnages noirs plus âgés, comme Roger Murtaugh dans la série des Armes fatales (interprété par Danny Glover) ou le sergent Al Powell dans Die Hard (1988). Au contact de héros blancs, des représentants des forces de l'ordre au comportement intempestif, ces personnages noirs plus âgés retrouvaient une forme de masculinité noire assertive sur le plan familial et professionnel, mais désexualisée à l'écran (Rehling 59). La compétence de Belle au volant, l'incapacité d'Andy Washburn à conduire et à impressionner son ancienne compagne, et sa volonté de revêtir le costume d'un immigrant cubain peuvent laisser penser que l'impuissance ethnique et raciale des buddy movies interraciaux des années 1980 a été transposée sur le personnage blanc dans New York Taxi. Il faut toutefois ne pas oublier que le film est une comédie, genre cinématographique qui permet précisément de bouleverser dans le courant du film les représentations de la féminité et de la masculinité, à condition qu'elles soient rétablies à la fin du film. Au début du long métrage, la masculinité blanche n'est donc que momentanément défaillante et l'objet du film est de la rétablir par le concours d'un personnage féminin noir.

Le film minore l'appartenance raciale du personnage interprété par Queen Latifah. Seule trois références indirectes en font état. Au début du film, on découvre Belle Williams dans la longue file d'attente d'hommes ethniques et non-blancs qui espèrent, comme elle, retirer leur licence de taxi. Dans la première scène dans laquelle on la voit conduire la Ford Crown à toute vitesse vers l'aéroport, elle dit au client blanc qui monte dans son taxi : «I don't usually stop for white guys, my way of balancing the universe ». Si elle se place ainsi, en tant que femme noire, sur une échelle de hiérarchie sociale, sexuelle et raciale, elle ne prendra dans le film que deux clients, tous les deux blancs. Enfin, lorsque le personnage d'Andy Washburn l'amène au domicile de sa mère alcoolique, cette dernière fait une allusion à la couleur de peau de Belle, se méprenant sur la relation potentielle qu'elle pourrait entretenir avec son fils, citant le titre du film de Spike Lee qui traite de relations interraciales («Nice catch, Jungle Fever»). Belle coupe alors aussitôt court à tout quiproquo. Les allusions à l'appartenance raciale de Belle sont donc clairsemées dans le film et ne focalisent pas l'attention sur une culture noire ou un univers racial propre au personnage. Pour reprendre l'expression d'Ed Guerrero, Belle est «culturellement différencié[e] par la simple couleur de peau » de l'actrice qui l'interprète à l'écran.

24 En cela, l'intervention du personnage de Queen Latifah dans le film s'apparente à celle de ce personnage stéréotypé noir qui naît dans les années 1990 et dont l'objectif narratif était de faire usage de sa spécificité pour aider le personnage masculin blanc à avancer dans sa mission, quelle qu'elle soit. Plusieurs auteurs, comme Krin Gabbard, illustrent ce stéréotype évoquant les personnages noirs de Ghost (1990), The Green Mile (1999) ou The Legend of Bagger Vance (2000). Dans ces black angel films, les pouvoirs surnaturels de ces personnages leur permettent d'aider les personnages masculins blancs à parvenir à une certaine forme de sérénité et de plénitude (144-145). Thomas Di Piero note que ces personnages stéréotypés ne possèdent pas nécessairement de pouvoirs spirituels et propose à cet effet une analyse des films Grand Canyon (1991) et White Men Can't Jump (1992) (210-211), reprise par Nicola Rehling :

Even if these black helpers lack spiritual powers, it has become a common motif for African-Americans to tell the white male who he is (59).

On comprend mieux dès lors la lecture très acerbe du critique du New York Times : 
[Queen Latifah's] bursting ethnicity somehow liberates Mr. Fallon's character [...]. By the end of the film, Washburn has been transformed into a dare-devil driver in his own right [...]. que l'on comprend le rôle d'agent de l'autorité paternelle que Belle est amené à jouer dans le film. Si ce personnage est très largement entouré d'hommes dans les scènes d'ouverture du film, sa maîtrise de la vitesse est également justifiée, plus tard dans le film, par la forte influence d'une figure paternelle dans l'enfance du personnage. C'est un phénomène que l'on retrouve chez les héroïnes d'action contemporaines. La mise en œuvre de leur action se manifeste sous le contrôle d'une présence masculine et paternelle qui est à l'initiative de la violence féminine que celle-ci ait pour but de la défendre, de la protéger, de la venger. Comme l'indique le monologue d'ouverture de Charlie dans Charlie's Angels cité précédemment, ces trois femmes travaillent pour lui. C'est bien ce fantomatique Charlie qui dérange le quotidien des trois héroïnes pour leur confier des missions à haut risque. De même, aussi spectaculaire que soit la scène d'ouverture de Lara Croft : Tomb Raider, c'est également un courrier retrouvé de son père, grand archéologue disparu, qui la guide à travers le monde sur les traces d'un trésor convoité par des ennemis lourdement armés. Enfin, c'est Bill dans Kill Bill qui motive la haine et le désir de vengeance de La Mariée. Jeffrey Brown utilise les travaux de Julia Kristeva pour théoriser le fait que ces héroïnes sont le produit d'une autorité paternelle :

Kristeva argues that the semiotic process involved with an individual's contact with authority can be contrasted between the alignment of the abject (e.g. bodily wastes) with maternal authority and the association of proper social regulation with paternal law. «Maternal authority is the trustee of that mapping of the self's clean and proper body, » writes Kristeva. «It is distinguished from paternal laws within which, with the phallic phase and acquisition of language, the destiny of man will take shape " [...]. Through her father, or father figures, the action heroine is made over in his image, and she is indoctrinated into the masculine realm of The Law. More than merely being masculinized through her aggressive behaviors, her muscular body, or her « manly » assumption of power, the action heroine becomes a dutiful subject and agent of patriarchal authority (78-79). ${ }^{6}$

Bien que l'action de Belle dans New York Taxi soit moins démonstrative que dans les films précédemment cités puisqu'elle se limite à la vitesse, on y retrouve cette filiation à une figure paternelle. Les spectateurs apprennent en effet plus tard dans le film que Belle tient son expertise de la mécanique, son goût pour la vitesse et sa maîtrise du volant de son grand-père, avec qui elle écoutait la retransmission des courses de Nascar. A l'inverse, la maladresse d'Andy Washburn au volant est due à un traumatisme de l'enfance, raconté par sa mère alcoolique dans une scène du film. Le père d'Andy l'aurait assis au volant de la voiture familiale à l'âge de 6 ans et l'aurait mis devant des responsabilités de chef de famille qu'à cet âge, il ne pouvait pas assumer (« You better steer or you'll kill the whole family »). Les deux protagonistes partagent en la matière un point commun : c'est une figure paternelle qui explique la maîtrise et l'expertise de l'une, et la maladresse de l'autre. Il manque donc une étape dans la construction de la masculinité d'Andy Washburn - le passage du garçon à l'homme - que Belle Williams a pour fonction narrative de combler, par le transfert de ses compétences au volant et de sa maîtrise de la vitesse.

C'est dans l'intimité du garage de Belle Williams que cette dernière tente d'amener Andy au-delà de son traumatisme. Le ressort comique et l'inversion des genres de la 
comédie sont maintenus puisque ce n'est qu'en chantant les paroles de «This Will Be an Everlasting Love " de Natalie Cole qu'Andy finit par maitriser son angoisse et conduire la voiture de Belle. Il opte donc pour cette chanson d'amour très populaire de 1975 et rejette, dès les premières paroles, le rythme endiablé et fortement racialisé que Belle lui propose, de la chanson sortie en 2001, «Pass the Courvoisier» («Don't this shit make a nigga wanna jump jump »), chanté par les rappeurs noirs Busta Rhymes et P. Diddy. La chanson est bien trop éloignée de l'univers culturel dans lequel il a grandi, comme il le reconnait lui-même. Même si Natalie Cole est elle aussi une chanteuse noire, Andy admet être plus à l'aise pour conduire en écoutant cette chanson dont il connaît parfaitement bien les paroles, puisqu'il avait l'habitude de les chanter devant son miroir quand il était petit, comme il finit par l'avouer. Sous couvert de proposer un ressort comique dans la construction de cette masculinité blanche moins virile, comme le permet le genre cinématographique de la comédie, le personnage, en s'appropriant une forme de familiarité avec la chanson de Natalie Cole, rejette la culture de la masculinité ethnique et raciale de la première chanson, «Pass the Courvoisier ». C'est donc en hurlant les paroles de la chanson, en tentant d'imiter les vocalises de Nathalie Cole, qu'Andy parvient à conduire aux côtés de Belle Williams qui chaperonne cette masculinité blanche en devenir. A la fin du film, c'est également en fredonnant la chanson de Nathalie Cole qu'Andy parvient à conduire la Ford Crown de Belle à toute vitesse pour lui sauver la vie. Cette scène, qui ne figure pas dans le film français, rétablit les représentations de genre du film au même titre qu'elle réhabilite la masculinité blanche du film. ${ }^{7}$ En 2003, dans Bringing Down the House, Queen Latifah (Charlene) occupait des fonctions similaires aux côtés de l'acteur blanc Steve Martin. Dans une scène du film, sur une chanson de Barry White, le personnage qu'interprète Queen Latifah apprend à celui incarné par Steve Martin (Peter) à faire ressortir une masculinité sexuelle moins sensible et plus agressive. A l'issue de cette comédie, grâce au concours de Charlene, Peter finissait par retrouver son épouse, menant Nicola Rehling à la conclusion suivante : «[In] Bringing Down the House (2003), Steve Martin learns to overcome his fastidious middle-class whiteness through his contact with female rap artist Queen Latifah» (177). Comme dans Bringing Down the House, la féminité noire de New York Taxi n'est qu'un outil libérateur visant surtout à construire la masculinité blanche, comme l'étaient les personnages masculins noirs des black angel films de la décennie précédente. Bien qu'elle soit sujet de l'action dans le film, Belle Williams reste un objet pour le sujet principal du récit, la masculinité blanche d'Andy Washburn.

C'est en cela que New York Taxi peut sembler différer du film français Taxi: le discours de la francité du film français Taxi, qui n'exclut pas pour autant une construction de la masculinité, est transposé dans la version américaine en discours de la masculinité blanche. Cette «traduction en langage hollywoodien » (Moine 2007) implique l'ajout de scènes dans le remake américain qui vont permettre de finaliser la transformation de la masculinité blanche à l'écran, en détruisant l'image filmique de la femme qui maîtrise la vitesse au volant. 


\section{La vitesse et l'occupation de l'espace urbain : du discours de la francité au discours de genre} deux personnages masculins qui habitent des quartiers très différents de la ville de Marseille, ces différences finissent par s'effacer pour déjouer le gang de braqueurs allemands et mettre à mal la menace étrangère qui pèse sur l'identité et l'économie française. Les autres épisodes de la saga reprendront cette opposition entre les deux héros français et les «méchants » étrangers, des Japonais dans Taxi 2, des Chinois dans Taxi 3 et des Belges dans Taxi 4. La nécessité de protéger l'identité française de la menace étrangère constitue donc une des thématiques centrales de la trame des films. Rappelons en effet qu'à la fin de Taxi, c'est bien pour le compte de la police nationale que Daniel Morales intègre une course de Grand Prix, et qu'à la fin de Taxi 2, il se retrouve sur les Champs Elysées au volant de son taxi lors du défilé du 14 juillet. anodin. Elle participe au «modèle » mis en place par Luc Besson, le scénariste de Taxi : un acteur d'origine algérienne, Samy Naceri, interprète un personnage, Daniel Morales, dont «le prénom laisse clairement penser que les origines du héros sont sinon gauloises, tout au moins européennes" (Gaertner 2005, 193). Aucune référence explicite aux origines algériennes de l'acteur n'est faite dans le film Taxi (McGonagle 135). Pour Alec G. Hargreaves, « l'effacement » des origines ethniques de l'acteur serait lié au genre du film, une comédie de divertissement « qui ne privilégie que des scènes de poursuites en voiture à grande vitesse » (Tarr 168). Hargreaves distingue ainsi les films comme Taxi, des films dits « de banlieue », destinés à un public plus ciblé, comme La Haine (Mathieu Kassovitz, 1995) ou Ma 6-té va crack-er (Jean-François Richet, 1997) dont les thématiques reposent sur la «fracture sociale » et les «clivages ethniques » (Hargreaves), mais pas nécessairement sur l'action et le divertissement.

Sur les origines ethniques de l'acteur à l'affiche de Taxi, Joseph McGonagle parle de l'identité «énigmatique » de Daniel Morales (135). De son côté, Julien Gaetner fait référence à une "étrangeté gommée", mais il souligne que cette dernière est particulièrement complexe dans un film où la «confusion culturelle" est "pertinemment travaillée » par son scénariste, Luc Besson (Gaertner 2005, 192-193). Si, en effet, aucun marqueur des origines ethniques de Daniel Morales n'est révélé dans la saga, la scène de Taxi 2 dans laquelle le personnage principal rencontre le père de son amie, général de l'armée française, suggère que les origines algériennes de l'acteur sont implicitement pointées du doigt par les allusions d'autres personnages à l'instar du commissaire Gibert dans Taxi (Bernard Farcy), dépeint lui aussi comme un ringard. Il n'est pas anodin que ces personnages démodés incarnent des figures de l'autorité et de l'ordre (un général de l'armée française et un commissaire de police). Si cet élément a été perçu comme un moyen de satisfaire une partie du public auquel le film s'adressait (Gaertner 2007, 167), on peut aussi envisager que ces personnages, auxquels les spectateurs ne peuvent s'identifier, servent de bornes au cadre identitaire de genre et de race dans lequel le modèle "Daniel Morales/Samy Naceri » évolue. La vitesse que déploie le personnage de Daniel Morales/Sami Nacery est mise au service de la protection de l'identité nationale, identité que Luc Besson modernise, masculinise et ethnicise. 
33 La masculinité ethnique dans Taxi diffère de celle mise en œuvre dans les films précédemment cités comme La Haine. À partir des travaux de Ginette Vincendeau, Carrie Tarr montre que la performance de la masculinité dans les films de «banlieues » des années 1990 sert de palliatif à une exclusion sociale sur laquelle les personnages de ces films n'ont aucune emprise (Tarr 111). Daniel Morales n'est pas le même personnage que celui que l'on peut trouver dans La Haine ou Ma 6-té va crack-er. Au contraire, au début de Taxi, Daniel passe d'un emploi à un autre, du statut d'employé à son propre compte, et c'est dans le hangar dans lequel il vit seul qu'on le voit séduire Lilly Bertineau (Marion Cotillard) et tenter vainement de consommer leur relation. On est donc loin de l'univers cloisonné des banlieues où les protagonistes des films végètent.

34 La défense de la francité sur laquelle la comédie d'action repose, recourt à une masculinité suffisamment forte et puissante pour contrer la menace étrangère. Cette masculinité est donc indissociable du véhicule de Daniel Morales, la Peugeot 406 trafiquée, de sa vitesse et de sa puissance, toutes deux opposées à celles des BMW allemandes. Les remarques avec lesquelles Daniel nargue les braqueurs allemands mêlent des références à l'économie automobile allemande à un discours dans lequel il féminise les chauffeurs teutons («Alors, les Bavaroises, ça va? Toujours en tracteurs ?»). De même, la présence de Daniel Morales aux côtés de l'inspecteur Emilien Coutant-Kerbalec/Frédéric Diefenthal doit permettre à ce dernier de séduire Pétra, sa collègue d'origine allemande. A juste titre, Raphaëlle Moine décrit Pétra (Emma Sjöberg) comme " piment érotique ", " contrepoids comique à la masculinité défaillante d'Émilien » et « l'équipe de bras cassés du commissariat marseillais » (Moine 2010, 85). Comme Lily Bertineau, ce second personnage féminin intègre comme accessoire le discours de la masculinité et le discours national du film, et met là aussi en lien la maîtrise du volant et de la vitesse, et la conquête du personnage féminin, exclu de la notion de vitesse. La poursuite finale de Taxi est d'ailleurs dépeinte comme une compétition franco-allemande, exclusivement masculine. Taxi corrèle la défense de la francité, au cœur de la trame du film, la maîtrise de la vitesse et la capacité de Daniel Morales à consommer sa relation avec Lilly Bertineau avant la fin du film. ${ }^{8}$ C'est chose faite, puisqu'après avoir déjoué le gang de braqueurs allemands et avant de recevoir une médaille pour les services qu'il a rendus à la communauté marseillaise, Daniel Morales satisfait sa compagne dans un placard de la mairie de Marseille.

La comparaison entre le film Taxi et son remake américain met donc en exergue une utilisation différente de la vitesse. En effet, là où Taxi envisage la vitesse dans un discours d'intégration ethnique, sans pour autant écarter la notion de masculinité, New York Taxi donne l'apparence d'intégrer les personnages féminins à la vitesse, mais se focalise en réalité sur la réhabilitation de la masculinité blanche, laissant volontairement de côté la dimension interraciale du duo constitué par les protagonistes principaux.

36 Si le remake reproduit la poursuite finale, et la façon dont les braqueurs/braqueuses sont immobilisé(e)s sur un tronçon d'autoroute, le remake ajoute des éléments qui ne sont pas dans la version française. L'analyse de ces deux ajouts narratifs et visuels illustre la focalisation du film sur la masculinité blanche et sur sa réappropriation de la vitesse. En effet, dans la version américaine, Andy Washburn sauve deux personnages féminins tour à tour : le lieutenant Robbins, dans un premier temps et Belle Williams dans un second. Même s'il n'est pas au volant, c'est le donneur d'ordre de la scène 
spectaculaire dans laquelle il récupère le lieutenant Robbins, la faisant passer d'une voiture à l'autre, alors que les deux véhicules filent à toute vitesse sur l'autoroute, au milieu d'autres voitures. Il fait alors preuve d'intelligence, de courage, de force et est plus actif dans le déroulement des étapes du récit qu'il ne l'était au début du film où il semblait subir l'action ou en être écarté. Sa maîtrise de la vitesse intervient après avoir déjoué les braqueuses. En effet, sa marche arrière à toute allure pour amener Belle Williams, blessée par balle, à l'hôpital est parfaitement contrôlée et le fait qu'il pénètre en taxi dans le hall de l'hôpital est résolument voulu, dans un contraste appuyé par rapport à sa confusion initiale. Ainsi, dans la version américaine, on voit le personnage masculin blanc maitriser la vitesse et le volant et se réapproprier l'espace urbain de manière désinhibée (Cahn 279). On passe donc d'un personnage qui peine à sortir d'un créneau la voiture de sa mère, une Daewoo Lanos, à un personnage qui maîtrise la Ford Crown, une voiture puissante, qui est également celle de la police et des taxis newyorkais, professions avant tout masculines, emblématiques de la maîtrise de l'espace et de l'ordre urbain.

Ce réinvestissement spatial est mis en opposition avec la façon dont le personnage de Vanessa est filmé, une fois coincée sur le tronçon de l'autoroute. La scène contraste avec celle, un peu plus tôt dans le film, dans laquelle la braqueuse brésilienne opère une fouille corporelle très suggestive sur le lieutenant Robbins, sous les yeux de la police impuissante. Les personnages masculins de la scène apprécient ce spectacle homoérotique, alors qu'il semble étrange à Belle et inutile au lieutenant que le subit. On comprend mieux cette scène si on la met en parallèle avec celle dans laquelle Vanessa contraint Belle à freiner violemment pour éviter un groupe d'enfants. La voiture de Belle finit par emboutir une bouche à incendie. Un plan en plongée extrême montre le jet d'eau du poteau jaillir de dessous la voiture, par le moteur, telle une éjaculation. Et Belle de conclure: «Damn, I was on her ass, too.» Un plan inséré sur le visage de Vanessa jetant un regard furtif dans le rétroviseur, le sourire aux lèvres, souligne sa satisfaction. Ces deux allusions sexuelles associées au personnage de Vanessa sont donc empruntes d'une forme d'homo-érotisme lesbien dans laquelle Vanessa exerce un pouvoir sexuel dominant sur le corps féminin (celui du lieutenant Robbins et par extension sur la voiture de Belle). Toutefois, à la fin du film, coincé sur le tronçon, ce personnage est privé de ce pouvoir. Le plan sur les hauts talons de la braqueuse, coincée sur le tronçon, devant le vide souligne son immobilité. Le plan est alterné à un autre en contre-plongée extrême, laissant imaginer l'entre-jambe de Vanessa, vêtue d'une mini-jupe. Au fur et à mesure que le personnage comprend qu'il est piégé les plans se resserrent pour ne laisser à l'écran qu'un personnage féminin, visible du haut de la poitrine, sans plus aucune référence aux talons aiguilles, au corps dénudé et à sa maitrise du volant. La colère hystérique du braqueur allemand au milieu de ses complices à la fin du film Taxi, est remplacée dans le remake par le mutisme et l'isolement visuel de la braqueuse brésilienne. Sans sa voiture, et surtout la vitesse qu'elle peut déchaîner dans les rues de New York, le personnage est enfermé dans un espace restreint tant sur le plan narratif (le tronçon d'autoroute) et sur le plan visuel (le cadre resserré du plan de la caméra), à l'inverse du personnage d'Andy Washburn, qui lance sa Ford à toute allure en marche arrière vers l'hôpital. Privé de sa vitesse, le personnage de Vanessa est progressivement déséroticisé et réduit à l'impuissance, dans un renversement avec le personnage masculin qui lui retrouve toute sa virilité. En effet, à la fin du film, le personnage masculin blanc du film s'est réapproprié son autorité sur 
les personnages féminins, sur la voiture américaine, autant que sur son occupation de l'espace urbain.

\section{Conclusion}

Bien que plus actives que les personnages féminins de Taxi, les personnages interprétés par Queen Latifah et le mannequin brésilien Giselle Bündchen n'en restent pas moins, chacune à leur façon, des accessoires à la construction du discours de la masculinité blanche mis en œuvre dans le remake américain. Le parallèle crée entre le personnage de Queen Latifah et celui de Giselle Bündchen, à travers la maîtrise de la vitesse, occulte littéralement la question raciale, au profit d'un discours axé sur le genre, et en particulier sur la masculinité. Comme dans Taxi, la vitesse dans New York Taxi est un outil qui permet de construire la masculinité du protagoniste principal blanc, qui, malgré les apparences du buddy movie, reste l'objet narratif principal du film. Ainsi, si les personnages féminins maîtrisent momentanément la vitesse, ils n'en sont pas moins les sujets du film.

La série inspirée du film Taxi, Taxi Brooklyn, produit par Europa Corp, la société de production de Luc Besson, a été diffusée sur les chaînes NBC et TF1 au printemps 2014. Pour les besoins du renouvellement de la trame de Taxi, le peureux Emilien CoutantKerbelec et le maladroit Andy Washburn ont été remplacé par la rugueuse Cat Sullivan. La race et le genre de la distribution du film ont été inversés puisque l'actrice blanche américaine Chyler Leigh donne la réplique à l'acteur noir d'origine burkinabé, Jacky Ido, qui joue le rôle de Daniel Morales, devenu Léo Romba. Cette fois, le personnage féminin sait parfaitement bien conduire, mais son tempérament intempestif la rend incontrôlable, voire dangereuse, au volant. De manière très intéressante, la trame secondaire des épisodes repose sur le désir de vengeance de Cat Sullivan, déterminée à mettre derrière les barreaux les assassins de son père, un ancien officier de la police de New York. On retrouve donc dans la trame de la série télévisée cette figure paternelle qui hante l'héroïne d'action, comme le notaient Kristeva et Brown, et qui fait d'elle le sujet et l'agent d'une autorité paternelle, ici familiale et institutionnelle.

\section{BIBLIOGRAPHIE}

Brown, Jeffrey. Dangerous Curves: Action Heroines, Gender, Fetichism and Popular Culture. Jackson: University Press of Mississippi, 2011.

Cahn, Susan K. Coming on Strong. Gender and Sexuality in Twentieth-Century Women's Sport. Cambridge, Mass: Harvard University Press, 1994.

Choi, Precilla Y. L. Femininity and the Physically Active Woman. London : Routledge, 2000.

Dipiero, Thomas. White Men Aren't. Durham: Duke University Press, 2002. 
Gabbard, Krin. Black Magic: White Hollywood and African American Culture. New Brunswick, NJ: Rutgers University Press, 2004.

Gaertner Julien. « Aspects et représentations du personnage arabe dans le cinéma français, 1995-2005. Retour sur une décennie. » Confluences Méditerranée 4:55 (2005): 189-201.

--- « Le préjugé se vend bien : Arabes et Asiatiques dans le discours cinématographique français. » Migrations et société 19:109 (janvier-février 2007): 163-173.

Gough-Yates, Anna. “Angels in Chains? Feminism, Femininity and Consumer Culture in Charlie's Angels." In Action TV: Tough Guys, Smooth Operators and Foxy Chicks. Ed. Bill Osgerby and Anna Gough-Yates. London and New York : Routledge, 2001, 83-99.

Guerrero, Ed. "The Black Image in Protective Custody: Hollywood's Biracial Buddy Films of the Eighties.” In Black American Cinema. Ed. Ed Guerrero. New York : Routledge, 1993. 237-246.

Haggins, Bambi L. “Crossover Diva: Whoopi Goldberg and Persona Politics.” In The Persistence of Whiteness: Race and Contemporary Hollywood Cinema. Ed. Daniel Bernardi. Oxon, New York : Routledge. 315-343.

Halberstam, Judith. Female Masculinity. Durham : Duke University Press, 1998.

Holmlund, Chris. "Wham! Bam! Pam!: Pam Grier as Hot Action Babe and Cool Action Mama." In Screening Genders. Ed. KrinGabbard and William Luhr. New Brunswick, NJ : Rutgers University Press, 2008, 61-77.

Jeffords, Susan. Hard Bodies: Hollywood Masculinity in the Reagan Era. New Brunswick, NJ : Rutgers University Press, 1994.

--- "Can Masculinity Be Terminated." In Screening the Male: Exploring Masculinities in Hollywood Cinema. Ed. Steven Cohan and Ina Rae Hark. London : Routledge, 1993. 245-262.

Kehr, Dave. "Stop Thief! Arrest that Model!" New York Times October 6, 2004. Généré le 20 août 2014. <http://www.nytimes.com/2004/10/06/movies/06taxi.html?_r=0>.

Lichtenfeld, Eric. Action Speaks Louder: Violence, Spectacle and the American Action Movie. Middleton, CT : Wesleyan University Press, 2007.

Mask, Mia. Divas on Screen: Black Women in American Film. Chicago : University of Illinois Press, 2009.

McGonagle, Joseph. « Ça roule à Marseille? Représentations de l'ethnicité dans les films Taxi (Gérard Pirès, 1998, Gérard Krawczyk, 2000, 2003, 2007). » In Langues et media en Méditerranée. Ed. Abdenbi Lachkar. Paris : L'Harmattan, 2012. 133-138.

Moine, Raphaëlle. Remakes : Les films français à Hollywood [en ligne]. Paris : CNRS Éditions, 2007. Généré le 20 août 2014. Disponible sur Internet : <http://books.openedition.org/editionscnrs/ 698>.

---. Les femmes d'action au cinéma. Paris : Armand Colin, 2010.

Mulvey, Laura. "Visual Pleasure and Narrative Cinema." Screen 16:3 (1975) : 6-18.

Forman, Murray. “Boys Will Be Boys: David Cronenberg's Crash Course in Heavy Mettle.” in Ladies and Gentlemen, Boys and Girls: Gender in Film at the End of the Twentieth Century. Ed. Murray Pomerance. Albany : State University of New York Press, 2001, 109-127.

Neroni, Hilary. The Violent Woman : Femininity, Narrative and Violence in Contemporary American Cinema. Albany, NY : State University of New York Press, 2005. 
Notes de production, Twentieth Century Fox, New York Taxi. Généré le 20 août 2014. <http:// www.foxfrance.com/presse/newyorktaxi/doc/notes.pdf >.

O’Day, Marc. "Beauty in Motion: Gender, Spectacle and Action Babe Cinema." In Action and Adventure Cinema. Ed. Yvonne Tasker. London : Routledge, 2004. 201-218.

O'Brien, Harvey. Action Movies: The Cinema of Striking Back. London and New York : Wallflower Press, 2012.

“Queen Latifah Stars as Fastest Cabbie in Hilarious Movie Taxi.” Jet 15:106 (2004) : 60-64.

Rehling, Nicola. Extra-Ordinary Men : White Heterosexual Masculinity in Contemporary Popular Cinema. Lanham, MD : Lexington Books, 2009.

Romao, Tico. "Guns and Gas : Investigating the 1970s Car Chase Film". In Action and Adventure Cinema. Ed. Yvonne Tasker. London : Routledge, 2004. 130-152.

Sellier, Geneviève. La Nouvelle Vague, un cinéma au masculin singulier. Paris : Editions CNRS, 2005.

Sims, Yvonne. Women of Blaxploitation : How the Black Action Heroine Changed American Popular Culture. Jefferson, NC : McFarland, 2006.

Tarr, Carrie. "Masculinity and Exclusion in Post-1995 Beur and 'Banlieue' Films.” In The Trouble with Men : Masculinities in European and Hollywood Cinema. Ed. Phil Powrie. et al. London : Wallflower Press, 2004. 110-120.

---. Reframing Difference : Beur and banlieue Filmmaking in France. Manchester : Manchester University Press, 2005.

Tasker, Yvonne. Spectacular Bodies: Gender, Race and the Action Cinema. London : Routledge, 1993.

Yau, Esther, C. M. «Introduction ». In Hong Kong Cinema in a Borderless World. Ed. Esther C. M. Yau. Minneapolis : The University of Minnesota Press, 2001. 1-30.

\section{Filmographie}

Bringing Down The House, dir. Adam Shankman, Touchstone Pictures, 2003.

Charlie's Angels, dir. McG, Columbia Pictures, 2000.

Kill Bill-I, dir. Quentin Tarantino, Miramax Films,2003.

La Haine, dir. Mathieu Kassovitz, Studio Canal, 1995.

Lara Croft: Tomb Raider, dir. Simon West, Paramount Pictures, 2001.

Ma 6-té va crack-er, dir. Jean-François Richet, Actes prolétariens, Why Not Productions, 1997.

Matrix, dir. Andy et Lana Wachowski, Warner Bros. 1999.

New York Taxi, dir. Tim Story, Twentieth Century Fox, 2004.

Set it Off, dir. J. Gary Gray, New Line Cinema, 1996.

Taxi, dir. Gérard Pirès, EuropaCorp, ARP SélectionEuropaCorp, ARP SélectionEuropaCorp., ARP Sélection,1998.

Terminator, dir. James Cameron, Hemdale Film Corporation and Orion Pictures, 1984.

Terminator 2: Judgment Day, dir. James Cameron, Coralco Pictures, 1991. 


\section{NOTES}

1. Trois opus de la saga suivront, tous réalisés par Gérard Krawczyk, en 2000 (Taxi 2), en 2003 (Taxi 3,) et en 2007 (Taxi 4). Les quatre volets de la saga attireront entre 25 et 27 millions de spectateurs dans les salles en France, dont dix pour le second opus (McGonagle, 133; Gaetner 2005, 192).

2. Le remake américain est également sorti sous le titre Taxi, mais afin d'éviter la confusion, nous utiliserons le titre New York Taxi pour faire référence au film américain dans cet article.

3. Voir Raphaëlle Moine (2010) pour une analyse du personnage du lieutenant Ripley, interprétée par Sigourney Weaver, dans Alien, le huitième passager (Ridley Scott, 1979).

4. Comme évoqué précédemment, l'utilisation de la vitesse est au cœur de New York Taxi alors qu'elle ne constitue qu'une forme de manifestation de l'action spectaculaire d'autres films comme Charlie's Angels ou Lara Croft. Le premier exclut l'usage de la force physique. En effet, hormis les deux coups de poings assénés par deux des braqueuses brésiliennes qui assomment les deux protagonistes principaux, ces dernières tirent en tout et pour tout deux séries de coups de feu dans deux scènes différentes du film. L'une des balles atteint l'arme d'un policier qui lâche aussitôt cette dernière avant de se réfugier derrière sa voiture, l'autre touche Belle dans l'épaule, dans l'avant-dernière scène du film. La manifestation de l'action des braqueuses brésiliennes est écartée de tout contact physique avec les autres personnages du film. Hormis le fait que les braqueuses sont interprétées par des mannequins et non des actrices professionnelles, cette absence de combat physique dans le film met en exergue une distance qui les sépare des protagonistes principalement masculins qui se contentent de les regarder à distance, comme le montrent de nombreux plans alternés du film. On distinguera alors Vanessa des autres braqueuses du gang.

5. Il cite à cet effet le réalisateur noir Robert Townsend : « Hollywood is afraid that if you have more than one black person in a movie you have a black movie » (239).

6. Analysant principalement le personnage de Sydney Bristow de la série télévisée Alias, Jeffrey Brown constate que la prédominance de cette figure paternelle dans la vie des hérö̈nes d'action contemporaines est également associée à l'absence marquée de la mère (80).

7. Dans la dernière scène du film, Andy Washburn revêtit à nouveau un costume ethnicisant son américanité, celui d'un proxénète russe, mais cette fois-ci, le lieutenant Robbins, son ancienne compagne, joue le rôle de l'une de ses prostituées.

8. Les quatre opus du film suivent par ailleurs l'évolution du personnage de Daniel sous le spectre de sa relation amoureuse avec ce personnage féminin: du premier rendez-vous (Taxi), à la présentation de la famille (Taxi 2) jusqu'à la paternité (Taxi 4).

\section{RÉSUMÉS}

Le film Taxi (1998) met en scène l'acteur d'origine algérienne, Samy Naceri, dans le rôle d'un ancien chauffeur de taxi qui met ses compétences au volant au service de la police marseillaise pour déjouer un gang de braqueurs allemands. Le succès de la saga des films Taxi repose sur le nombre de scènes de poursuite de bolides vrombissants dans les rues de Marseille. Le remake du film, New York Taxi (2004), transforme les rôles du film français. L'as du volant est interprété par l'actrice noire américaine Queen Latifah et les braqueurs allemands deviennent des braqueuses brésiliennes, interprétés par des mannequins. Aussi, le discours de la francité, au cœur de la 
comédie d'action française, est transposé, dans la version américaine, vers un discours de la masculinité blanche. Dans cet article, nous nous intéresserons à la façon dont la maîtrise de la vitesse par les personnages féminins de New York Taxi participe à la transformation de la masculinité blanche mise en scène dans le film.

Taxi (1998) is a French action comedy telling the story of a former pizza deliveryman who becomes a taxi driver at the beginning of the film. He is forced into helping a police officer of Marseille to defeat a gang of German bank robbers. The success of the film and of its sequels is mainly due to car chases in the streets of the city of Marseille. Its remake, New York Taxi (2004), gave the leading roles to the African American actress Queen Latifah and the white situation comedian Jimmy Fallon, shifting the male bonding relationship of the French version to an interracial pair. This shift also implied that the French film's central focus on Frenchness is diverted, in the American remake, into a discourse on white masculinity. The aim of this article is to consider how the use of speed in the filmic construction of the female characters in New York Taxi (2004) is meant to construct the white masculinity of the film's central character.

\section{INDEX}

Mots-clés : film d'action, vitesse motorisée, masculinité, femmes d'action, Queen Latifah, remake transnational, Taxi/New York Taxi

Keywords : action films, high-speed cars, masculinity, action heroines, Queen Latifah, transnational remake films, Taxi/New York Taxi

\section{AUTEUR}

\section{HÉLÈNE CHARLERY}

Maître de conférences

Université Toulouse 2-Jean Jaurès

helene.charlery@free.fr 